JoAnna PęTEla (Wrocław)

\title{
EXTINCTION IN NONAUTONOMOUS KOLMOGOROV SYSTEMS
}

Abstract. We consider nonautonomous competitive Kolmogorov systems, which are generalizations of the classical Lotka-Volterra competition model. Applying Ahmad and Lazer's definitions of lower and upper averages of a function, we give an average condition which guarantees that all but one of the species are driven to extinction.

1. Introduction. In recent years investigation of population dynamics has developed rapidly. One of the famous models for dynamics of a population is the Lotka-Volterra competition system

$$
u_{i}^{\prime}(t)=u_{i}(t)\left(a_{i}(t)-\sum_{j=1}^{N} b_{i j}(t) u_{j}(t)\right), \quad i=1, \ldots, N .
$$

where $a_{i}, b_{i j}:[0, \infty) \rightarrow(0, \infty)$. The model $(\overline{\mathrm{LV}})$ has been studied by many authors. They obtained a lot of results dealing with persistence and global attractivity. Gopalsamy [5], 6] and Tineo and Alvarez [9] showed that if

$$
a_{i L}>\sum_{\substack{j=1 \\ j \neq i}}^{N} \frac{b_{i j M} a_{j M}}{b_{j j L}} \quad \text { for } i=1, \ldots, N
$$

where $g_{L}$ (resp. $g_{M}$ ) denotes the infimum (resp. supremum) of the function $g$, then system $(\overline{\mathrm{LV}})$ is persistent and globally attractive. To be more precise, Gopalsamy proved that in the almost periodic case the conditions GAT

2010 Mathematics Subject Classification: Primary 34D05; Secondary 34C12, 34C29, 34D23, 92D40.

Key words and phrases: Kolmogorov system, Lotka-Volterra system, upper average, lower average, logistic equation, global attractivity. 
together with the conditions

$$
b_{j j L}>\sum_{\substack{j=1 \\ j \neq i}}^{N} b_{i j L} \quad \text { for } i=1, \ldots, N
$$

imply persistence and global attractivity of $(\mathrm{LV})$. Then Alvarez and Tineo showed that we may drop assumption (D). Ahmad and Lazer [2] proved that persistence and global attractivity hold under the conditions

$$
m\left[a_{i}\right]>\sum_{\substack{j=1 \\ j \neq i}}^{N} \frac{b_{i j M} M\left[a_{j}\right]}{b_{j j L}} \quad \text { for } i=1, \ldots, N
$$

where

$$
m[g]=\liminf _{t-s \rightarrow \infty} \frac{1}{t-s} \int_{s}^{t} g(\tau) d \tau, \quad M[g]=\limsup _{t-s \rightarrow \infty} \frac{1}{t-s} \int_{s}^{t} g(\tau) d \tau .
$$

In [1] and [4] a nonautonomous logistic equation

$$
u_{0}^{\prime}(t)=u_{0}(t)\left(a(t)-b(t) u_{0}(t)\right), \quad t \in \mathbb{R},
$$

is considered. It is well known that an autonomous logistic equation

$$
u^{\prime}=u(b-a u)
$$

with $a, b>0$ has a global attractor on $(0, \infty)$ at the carrying capacity $x=b / a$. Ahmad [1] and Coleman [4] showed that in the nonautonomous equation $(\mathrm{L})$ the role of the globally attracting carrying capacity of the autonomous equation is played by a well defined canonical solution $u_{i}^{*}(t)$ to which all other solutions converge.

LEMma 1 (Ahmad and Coleman [1, 4]). Assume that in (L) the functions $a(t), b(t)$ are continuous and bounded above and below by positive constants. Then equation (L) has a unique solution $u^{*}$ which is bounded above and below by positive reals for all $t$.

We should emphasize that in Lemma 1 we assume that the solution of equation ( $\mathrm{L}$ ) is bounded and bounded away from zero on the whole of $\mathbb{R}$.

Lemma 2 (Coleman [4]). Suppose that in (L) the functions $a(t), b(t)$ are continuous and bounded above and below by positive constants. If $u(t), v(t)$ are positive solutions of $(\mathrm{L})$ then $u(t)-v(t) \rightarrow 0$ as $t \rightarrow \infty$.

Thus $u(t), v(t) \rightarrow u^{*}$. At the same time Francisco Montes de Oca and Mary Lou Zeeman dealt with extinction. They considered a competing system $(\mathrm{LV})$, where $a_{i}, b_{i j}: \mathbb{R} \rightarrow(0, \infty)$ are continuous functions bounded by positive reals. In [7] they gave algebraic criteria on the parameters which 
guarantee that all but one of the species are driven to extinction; namely, for each $k>1$ there exists $i_{k}<k$ such that for any $j \leq k$,

$$
a_{k M} b_{i_{k} j M}<b_{i_{k} L} a_{k j L} .
$$

They proved that under condition $(\mathrm{E})$ the species $u_{2}, \ldots, u_{N}$ are driven to extinction whilst $u_{1}$ stabilizes at the unique bounded solution $u_{1}^{*}$ of the logistic equation on the $u_{1}$ axis. Moreover, they showed convergence of trajectories to $u_{1}^{*}$. Further, they gave a geometric interpretation of (E). In [3], Ahmad and Montes de Oca studied the $T$-periodic system $(\overline{\mathrm{LV}})$, i.e., the coefficients of (LV) are continuous and periodic with a common period $T$. They obtained the same result as in [7] under the assumption that for each $k>1$, there exists $i_{k}<k$ such that for any $j \leq k$,

$$
\bar{a}_{k} b_{i_{k} j}(t)-\bar{a}_{i_{k}} b_{k j}(t)<0
$$

where

$$
\bar{a}_{i}=\frac{1}{T} \int_{0}^{T} a_{i}(\tau) d \tau>0, \quad i=1, \ldots, N .
$$

In this paper we extend some of the above results to the case of $N$-species nonautonomous competitive Kolmogorov systems. We consider a system

$$
u_{i}^{\prime}=u_{i} f_{i}(t, u)
$$

on the nonnegative cone

$$
C=\left\{u=\left(u_{1}, \ldots, u_{N}\right): u_{i} \geq 0,1 \leq i \leq N\right\},
$$

where

(1) $f=\left(f_{1}, \ldots, f_{N}\right):[0, \infty) \times C \rightarrow \mathbb{R}^{N}$ is continuous together with its first derivatives $\partial f_{i} / \partial u_{j}$,

(2) for each compact set $\tilde{C} \subset C, \frac{\partial f_{i}}{\partial u_{j}}(t, u)$ are bounded and uniformly continuous on $[0, \infty) \times \tilde{C}$,

(3) there exist $a_{i}^{(1)}, a_{i}^{(2)}>0$ such that $a_{i}^{(1)} \leq f_{i}(t, 0, \ldots, 0) \leq a_{i}^{(2)}$ for all $t \geq 0$ and $1 \leq i \leq N$,

(4) $\frac{\partial f_{i}}{\partial u_{j}}(t, u) \leq 0$ for all $t \geq 0, u \in C$, and $i, j=1, \ldots, N$,

(5) there exist $b_{i i}^{(1)}>0$ such that $\frac{\partial f_{i}}{\partial u_{i}}(t, u) \leq-b_{i i}^{(1)}$ for all $t \geq 0, u \in C$, and $i=1, \ldots, N$.

We give a condition (Theorem 1) which implies that all but one of the species are driven to extinction. Moreover, if $U_{1}^{*}(t)$ is the solution of the equation

$$
U_{1}^{\prime}(t)=U_{1}^{\prime}(t) f_{1}\left(t, u_{1}(t), \ldots, u_{N}(t)\right)
$$

and $u(t)=\left(u_{1}(t), \ldots, u_{N}(t)\right)$ is the positive solution of (1.1) then $u_{1}(t)-$ $U_{1}^{*}(t) \rightarrow 0$ as $t \rightarrow \infty$ (Theorem 2). 
2. Preliminaries. We start by proving the following

Lemma 3. If $u:\left[t_{0}, \tau_{\max }\right) \rightarrow C, t_{0} \geq 0$, is a maximally defined solution of (1.1) such that $u_{i}(0)>0$ for $i=1, \ldots, N$, then $u_{i}(t)>0$ for $i=1, \ldots, N$ and $t \in\left[t_{0}, \tau_{\max }\right)$,

Proof. By 1.1,

$u_{i}(t)=u_{i}(0) \exp \left(\int_{t_{0}}^{t} f_{i}\left(s, u_{1}(s), \ldots, u_{N}(s)\right) d s\right)>0, \quad t \in\left[t_{0}, \tau_{\max }\right)$.

Lemma 4. If $u:\left[t_{0}, \tau_{\max }\right) \rightarrow C, t_{0} \geq 0$, is a maximally defined solution of (1.1) such that $u_{i}(0)>0$ for $i=1, \ldots, N$, then

(i) $\tau_{\max }=\infty$,

(ii) $\lim \sup _{t \rightarrow \infty} u_{i}(t) \leq a_{i}^{(2)} / b_{i i}^{(1)}$ for $i=1, \ldots, N$.

Proof. (i) Note that by assumption (4),

$$
u_{i}(t) \leq u_{i}\left(t_{0}\right) \exp \left(\int_{t_{0}}^{\tau_{\max }} f_{i}(s, 0, \ldots, 0) d s\right) .
$$

Hence we see that $\tau_{\max }=\infty$.

(ii) By assumptions (3), (4) and (5),

$$
u_{i}^{\prime} \leq u_{i}\left(a_{i}^{(2)}-b_{i i}^{(1)} u_{i}(t)\right) .
$$

Let $x_{i}(t)$ be the solution of the logistic equation

$$
x_{i}^{\prime}=x_{i}\left(a_{i}^{(2)}-b_{i i}^{(1)} x_{i}(t)\right)
$$

satisfying the initial condition $x_{i}\left(t_{0}\right)=u_{i}\left(t_{0}\right)$. Then by the comparison principle,

$$
u_{i}(t) \leq x_{i}(t) \quad \text { for } t \geq t_{0}, i=1, \ldots, N .
$$

For any positive solution $x_{i}(t)$ of 1.2 we have $\lim _{t \rightarrow \infty} x_{i}(t)=a_{i}^{(2)} / b_{i i}^{(1)}$ for $i=1, \ldots, N$. This yields the desired result.

Define

$$
\begin{aligned}
B & :=\left[0, a_{1}^{(2)} / b_{11}^{(1)}\right] \times \cdots \times\left[0, a_{N}^{(2)} / b_{N N}^{(1)}\right], \\
b_{i j}^{(2)} & :=-\inf \left\{\frac{\partial f_{i}}{\partial u_{j}}(t, x): t \geq 0, x \in B\right\} .
\end{aligned}
$$

Assumptions (2) and (4) guarantee that $0 \leq b_{i j}^{(2)}<\infty$. Further, define

$$
a^{(1)}:=\min \left\{a_{i}^{(1)}: i=1, \ldots, N\right\}, \quad b^{(2)}:=\max \left\{b_{i j}^{(2)}: i, j=1, \ldots, N\right\} .
$$


Lemma 5. There exists $\delta>0$ such that if $u(t)=\left(u_{1}(t), \ldots, u_{N}(t)\right)$ is a positive solution of (1.1) then

$$
\liminf _{t \rightarrow \infty} \sum_{i=1}^{N} u_{i}(t) \geq \delta \text {. }
$$

Proof. Let

$$
V(t):=\sum_{i=1}^{N} u_{i}(t)
$$

Then

$$
\begin{aligned}
V^{\prime}(t) & =\sum_{i=1}^{N} u_{i}^{\prime}(t)=\sum_{i=1}^{N} u_{i}(t) f_{i}\left(t, u_{1}(t), \ldots, u_{N}(t)\right) \\
& \geq V(t) \min \left\{f_{i}\left(t, u_{1}(t), \ldots, u_{N}(t)\right): 1 \leq i \leq N\right\} .
\end{aligned}
$$

We take $0 \leq \delta<a^{(1)} / b^{(2)}$ such that the set

$$
P_{\delta}=\left\{u \in C: \sum_{i=1}^{N} u_{i} \leq \delta\right\}
$$

is contained in $B$. We claim that $V^{\prime}(t)>0$ if $u(t)=\left(u_{1}(t), \ldots, u_{N}(t)\right) \in P_{\delta}$. Indeed, if $u(t) \in P_{\delta}$ then

$$
V^{\prime}(t) \geq V(t)\left[a^{(1)}-\sum_{j=1}^{N} b^{(2)} u_{j}(t)\right]=V(t)\left[a^{(1)}-b^{(2)} V(t)\right]>0 .
$$

We have thus proved that $V^{\prime}(t)>0$ as long as $(0<) V(t) \leq \delta$. Consequently, $\liminf _{t \rightarrow \infty} V(t) \geq \delta$. By the definition of $V$ we have $\liminf _{t \rightarrow \infty} \sum_{i=1}^{N} u_{i}(t)$ $\geq \delta$.

Define

$$
\begin{aligned}
B(\eta) & :=\left[0, a_{1}^{(2)} / b_{11}^{(1)}+\eta\right] \times \cdots \times\left[0, a_{N}^{(2)} / b_{N N}^{(1)}+\eta\right], \quad \eta \geq 0 \\
\beta_{i j}(\eta) & :=-\inf \left\{\frac{\partial f_{i}}{\partial u_{j}}(t, x): t \geq 0, x \in B(\eta)\right\}, \quad 1 \leq i, j \leq N .
\end{aligned}
$$

By assumptions (2), (4) and (5), $0 \leq \beta_{i j}(\eta)<\infty$.

LEMMA 6.

$$
\lim _{\eta \rightarrow 0^{+}} \beta_{i j}(\eta)=b_{i j}^{(2)} .
$$

In the proof of Lemma $6, u, v$ are elements of $C$, and $\|\cdot\|$ denotes the Euclidean norm in $\mathbb{R}^{N}$.

Proof of Lemma 6 . Notice that $\eta \mapsto \beta_{i j}(\eta)$ is nondecreasing. Hence the limit $\lim _{\eta \rightarrow 0^{+}} \beta_{i j}(\eta)$ exists, and $\lim _{\eta \rightarrow 0^{+}} \beta_{i j}(\eta) \geq b_{i j}^{(2)}$. Take $\varepsilon>0$. It suffices 
to prove that there exists $\vartheta>0$ such that for all $t \geq 0$ and $u \in B(\vartheta)$,

$$
-\frac{\partial f_{i}}{\partial u_{j}}(t, u)-b_{i j}^{(2)} \leq \varepsilon .
$$

By assumption (2), there exists $\eta>0$ such that if $\|u-v\|<\eta$ then

$$
\left|\frac{\partial f_{i}}{\partial u_{j}}(t, u)-\frac{\partial f_{i}}{\partial u_{j}}(t, v)\right|<\varepsilon, \quad u, v \in B(\eta), t \geq 0 .
$$

For each $u \in B(\eta / 2)$ there is $v \in B$ such that $\|u-v\| \leq \eta / 2<\eta$. Hence

$$
\frac{\partial f_{i}}{\partial u_{j}}(t, u)-\frac{\partial f_{i}}{\partial u_{j}}(t, v) \geq-\varepsilon, \quad t \geq 0, u \in B(\eta / 2) .
$$

Therefore

$$
-\frac{\partial f_{i}}{\partial u_{j}}(t, u)+\frac{\partial f_{i}}{\partial u_{j}}(t, v) \leq \varepsilon, \quad t \geq 0 .
$$

By (1.4), for all $t \geq 0, x \in B$,

Hence, by (1.6),

$$
\frac{\partial f_{i}}{\partial u_{j}}(t, x) \geq-b_{i j}^{(2)}
$$

$$
-\frac{\partial f_{i}}{\partial u_{j}}(t, u) \leq-\frac{\partial f_{i}}{\partial u_{j}}(t, v)+\varepsilon \leq b_{i j}^{(2)}+\varepsilon, \quad t \geq 0, u \in B(\eta / 2),
$$

which proves 1.5 .

We now define the lower and upper averages of a function $g$ which is continuous and bounded above and below on $[0, \infty)$. If $0<s<t$ we set

$$
A[g, t, s]:=\frac{1}{t-s} \int_{s}^{t} g(\tau) d \tau \text {. }
$$

The lower and upper averages of $g$ are defined by

$$
m[g]:=\liminf _{t-s \rightarrow \infty} A[g, t, s], \quad M[g]:=\limsup _{t-s \rightarrow \infty} A[g, t, s] .
$$

Notice that by assumption (3),

$$
a_{i}^{(1)} \leq \frac{1}{t-s} \int_{s}^{t} f_{i}(\tau, 0, \ldots, 0) d \tau \leq a_{i}^{(2)} \quad \text { for } 1 \leq i \leq N, 0<s<t .
$$

Hence

$$
a_{i}^{(1)} \leq m\left[f_{i}(\cdot, 0, \ldots, 0)\right] \leq M\left[f_{i}(\cdot, 0, \ldots, 0)\right] \leq a_{i}^{(2)} .
$$

In [8] we introduced average conditions for Kolmogorov systems

$$
m\left[f_{i}(\cdot, 0, \ldots, 0)\right]>\sum_{\substack{j=1 \\ j \neq i}}^{N} \frac{b_{i j}^{(2)} M\left[f_{j}(\cdot, 0, \ldots, 0)\right]}{b_{j j}^{(1)}} \quad \text { for } i=1, \ldots, N,
$$

which guarantee that system 1.1 is permanent and globally attractive. 
Define

$$
b_{i j}^{(1)}:=-\sup \left\{\frac{\partial f_{i}}{\partial u_{j}}(t, x): t \geq 0, x \in B\right\} .
$$

\section{Main theorem}

THEOREM 1. Assume that for every $k>1$ there exists $i_{k}<k$ such that for all $j \leq k$,

$$
\frac{M\left[f_{k}(\cdot, 0, \ldots, 0)\right]}{m\left[f_{i_{k}}(\cdot, 0, \ldots, 0)\right]}<\frac{b_{k j}^{(1)}}{b_{i_{k} j}^{(2)}}
$$

If $u=\left(u_{1}(t), \ldots, u_{N}(t)\right)$ is a positive solution of 1.1 then $u_{i}(t) \rightarrow 0$ as $t \rightarrow \infty$ for all $i=2, \ldots, N$.

The idea of the proof comes from 7 .

Proof. Let $u(t)=\left(u_{1}(t), \ldots, u_{N}(t)\right)$ be a positive solution of system (1.1). We argue by induction. First we show that $u_{N}(t) \rightarrow 0$ as $t \rightarrow \infty$. Let $i=i_{N}$ be given by inequality $\left(\mathrm{E}^{\prime}\right)$. By assumptions $(3)-(5)$ and the mean value theorem,

$$
u_{N}^{\prime}(t) \leq u_{N}(t)\left(f_{N}(t, 0, \ldots, 0)-\sum_{j=1}^{N} b_{N j}^{(1)} u_{j}(t)\right) .
$$

Fix $\eta>0$ such that

$$
\frac{M\left[f_{N}(t, 0, \ldots, 0)\right]}{m\left[f_{i}(t, 0, \ldots, 0)\right]}<\frac{b_{N j}^{(1)}}{\beta_{i j}(\eta)} .
$$

By Lemma 4(ii) there is $t_{1}>0$ such that $u(t) \in B(\eta)$ for $t>t_{1}$. Hence and by the mean value theorem,

$$
u_{i}^{\prime}(t) \geq u_{i}(t)\left(f_{i}(t, 0, \ldots, 0)-\sum_{j=1}^{N} \beta_{i j}(\eta) u_{j}(t)\right) \quad \text { for } t>t_{1} .
$$

By (1.9), we can choose $\alpha, \gamma>0$ such that for $j \leq N$,

$$
\frac{M\left[f_{N}(\cdot, 0, \ldots, 0)\right]}{m\left[f_{i}(\cdot, 0, \ldots, 0)\right]}<\frac{\alpha}{\gamma}<\frac{b_{N j}^{(1)}}{\beta_{i j}(\eta)} .
$$

Let

$$
V_{N}:=u_{i}^{-\alpha} u_{N}^{\gamma}
$$

Then

$$
\frac{d V_{N}}{d t}=V_{N}\left(-\alpha \frac{u_{i}^{\prime}(t)}{u_{i}(t)}+\gamma \frac{u_{N}^{\prime}(t)}{u_{N}(t)}\right)
$$


Therefore by 1.8 and 1.10 ,

$$
\begin{aligned}
\frac{d V_{N}}{d t} \leq & V_{N}\left(-\alpha\left(f_{i}(t, 0, \ldots, 0)-\sum_{j=1}^{N} \beta_{i j}(\eta) u_{j}(t)\right)\right. \\
& \left.+\gamma\left(f_{N}(t, 0, \ldots, 0)-\sum_{j=1}^{N} b_{N j}^{(1)} u_{j}(t)\right)\right) \\
= & V_{N}\left(\gamma f_{N}(t, 0, \ldots, 0)-\alpha f_{i}(t, 0, \ldots, 0)\right. \\
& \left.+\sum_{j=1}^{N} \gamma \beta_{i j}(\eta)\left(\frac{\alpha}{\gamma}-\frac{b_{N j}^{(1)}}{\beta_{i j}(\eta)}\right) u_{j}(t)\right) \text { for } t>t_{1} .
\end{aligned}
$$

By (1.11) we can choose $\zeta>0$ such that

$$
\frac{\alpha}{\gamma}-\frac{b_{N j}^{(1)}}{\beta_{i j}(\eta)}<-\zeta \quad \text { for } j \leq N .
$$

Hence

$$
\frac{d V_{N}}{d t} \leq V_{N}\left(\gamma f_{N}(t, 0, \ldots, 0)-\alpha f_{i}(t, 0, \ldots, 0)-\zeta \gamma \hat{\beta}_{i}(\eta) \sum_{j=1}^{N} u_{j}(t)\right)
$$

where $\hat{\beta}_{i}(\eta)=\min \left\{\beta_{i j}(\eta): j=1, \ldots, N\right\}$. By Lemma 5 , there exists $t_{2}>t_{1}$ such that

$$
\sum_{j=1}^{N} u_{j}(t)>\delta / 2 \quad \text { for } t>t_{2}
$$

Hence

$$
\frac{d V_{N}}{d t} \leq V_{N}\left(\gamma f_{N}(t, 0, \ldots, 0)-\alpha f_{i}(t, 0, \ldots, 0)-\xi\right) \quad \text { for } t>t_{2},
$$

where

$$
\xi=\zeta \gamma \beta_{i j}(\eta) \delta / 2>0
$$

Hence

$V_{N}(t) \leq V_{N}\left(t_{2}\right) \exp \left\{\int_{t_{2}}^{t}\left(\gamma f_{N}(\tau, 0, \ldots, 0)-\alpha f_{i}(\tau, 0, \ldots, 0)-\xi\right) d \tau\right\} \quad$ for $t>t_{2}$.

By the definition of $V_{N}$ we have

$$
\begin{aligned}
u_{N}(t)< & \left(u_{i}^{\alpha}(t) \frac{u_{N}^{\gamma}\left(t_{2}\right)}{u_{i}^{\alpha}\left(t_{2}\right)}\right)^{1 / \gamma} \\
& \times \exp \left\{\frac{1}{\gamma} \int_{t_{2}}^{t}\left(\gamma f_{N}(\tau, 0, \ldots, 0)-\alpha f_{i}(\tau, 0, \ldots, 0)-\xi\right) d \tau\right\}
\end{aligned}
$$


for $t>t_{2}$. By Lemma 4(i) there exists $R>0$ such that $u_{j}(t)<R$ for $j=1, \ldots, N$. Therefore

$$
u_{N}(t)<C \exp \left\{\frac{1}{\gamma} \int_{t_{2}}^{t}\left(\gamma f_{N}(\tau, 0, \ldots, 0)-\alpha f_{i}(\tau, 0, \ldots, 0)-\xi\right) d \tau\right\}
$$

for $t>t_{2}$, where

$$
C=\left(R^{\alpha} \frac{u_{N}^{\gamma}\left(t_{2}\right)}{u_{i}^{\alpha}\left(t_{2}\right)}\right)^{1 / \gamma} .
$$

Now we show that

$$
\lim _{t \rightarrow \infty} \int_{t_{2}}^{t}\left(\gamma f_{N}(\tau, 0, \ldots, 0)-\alpha f_{i}(\tau, 0, \ldots, 0)-\xi\right) d \tau=-\infty .
$$

By (1.11),

$$
\gamma M\left[f_{N}(t, 0, \ldots, 0)\right]-\alpha m\left[f_{i}(t, 0, \ldots, 0)\right]<0 .
$$

Since

$$
\begin{aligned}
& \limsup _{t-t_{2} \rightarrow \infty} \frac{1}{t-t_{2}} \int_{t_{2}}^{t}\left(\gamma f_{N}(t, 0, \ldots, 0)-\alpha f_{i}(t, 0, \ldots, 0)\right) d \tau \\
& \quad \leq \gamma \limsup _{t-t_{2} \rightarrow \infty} \frac{1}{t-t_{2}} \int_{t_{2}}^{t} f_{N}(t, 0, \ldots, 0) d \tau-\alpha \liminf _{t-t_{2} \rightarrow \infty} \frac{1}{t-t_{2}} \int_{t_{2}}^{t} f_{i}(t, 0, \ldots, 0) d \tau \\
& \quad=\gamma M[f(t, 0, \ldots, 0)]-\alpha m[f(t, 0, \ldots, 0)]<0 \quad \text { for } t>t_{2},
\end{aligned}
$$

it follows that

$$
\limsup _{t-t_{2} \rightarrow \infty} \int_{t_{2}}^{t}\left(\gamma f_{N}(\tau, 0, \ldots, 0)-\alpha f_{i}(\tau, 0, \ldots, 0)\right) d \tau=-\infty \quad \text { for } t>t_{2} .
$$

Therefore

$$
\lim _{t \rightarrow \infty} \int_{t_{2}}^{t}\left(\gamma f_{N}(\tau, 0, \ldots, 0)-\alpha f_{i}(\tau, 0, \ldots, 0)-\xi\right) d \tau=-\infty
$$

for $t>t_{2}$. Hence and by (1.14) it follows that $u_{N}(t) \rightarrow 0$ as $t \rightarrow \infty$.

Now we prove that for $1<p<N, u_{p}(t) \rightarrow 0$ as $t \rightarrow \infty$ under the assumption that for $p<j<N, u_{j}(t) \rightarrow 0$ as $t \rightarrow \infty$. Let $i=i_{p}$ be given by inequality $\left(\mathrm{E}^{\prime}\right)$. Fix $\eta>0$ such that

$$
\frac{M\left[f_{p}(\cdot, 0, \ldots, 0)\right]}{m\left[f_{i}(\cdot, 0, \ldots, 0)\right]}<\frac{b_{p j}^{(1)}}{\beta_{i j}(\eta)} .
$$


From assumption (4), (5) and by the mean value theorem,

$$
u_{p}^{\prime}(t) \leq u_{p}(t)\left(f_{p}(t, 0, \ldots, 0)-\sum_{j=1}^{N} b_{p j}^{(1)} u_{j}(t)\right) .
$$

Lemma 4(ii) implies the existence of $t_{1}>0$ such that $u(t) \in B(\eta)$ for $t>t_{1}$. Hence

$$
u_{i}^{\prime}(t) \geq u_{i}(t)\left(f_{i}(t, 0, \ldots, 0)-\sum_{j=1}^{N} \beta_{i j}(\eta) u_{j}(t)\right) \quad \text { for } t>t_{1} .
$$

By (1.17), we can choose $\lambda, \kappa>0$ such that for $j \leq p$,

$$
\frac{M\left[f_{p}(\cdot, 0, \ldots, 0)\right]}{m\left[f_{i}(\cdot, 0, \ldots, 0)\right]}<\frac{\lambda}{\kappa}<\frac{b_{p j}^{(1)}}{\beta_{i j}(\eta)} .
$$

Note that by (1.20) there exists $\varsigma>0$ such that

$$
\frac{\lambda}{\kappa}-\frac{b_{p j}^{(1)}}{\beta_{i j}(\eta)}<-\varsigma<0 \quad \text { for } j \leq p .
$$

Let

$$
V_{p}:=u_{i}^{-\lambda} u_{p}^{\kappa}
$$

Similarly to $1.12-1.13$ we get

$$
\begin{aligned}
\frac{d V_{p}}{d t} \leq V_{p}\left(\kappa f_{p}(t, 0, \ldots, 0)\right. & -\lambda f_{i}(t, 0, \ldots, 0) \\
& \left.-\rho \sum_{j=1}^{p} u_{j}(t)+\sum_{j=p+1}^{N}\left(\lambda \beta_{i j}(\eta)-\kappa b_{p j}^{(1)}\right) u_{j}(t)\right),
\end{aligned}
$$

where $\rho=\kappa \min \left\{\beta_{i j}(\eta): j=1, \ldots, N\right\} \varsigma>0$. Note that by Lemma 5 there exists $t_{2}>0$ such that

Hence

$$
\sum_{i=1}^{p} u_{i}(t)+\sum_{i=p+1}^{N} u_{i}(t)>\delta / 2 \quad \text { for } t>t_{2} .
$$

$$
\begin{aligned}
\frac{d V_{p}}{d t} \leq & V_{p}\left(\kappa f_{p}(t, 0, \ldots, 0)-\lambda f_{i}(t, 0, \ldots, 0)-\rho \delta / 2\right. \\
& \left.+\sum_{j=p+1}^{N}\left(\lambda \beta_{i j}(\eta)-\kappa b_{p j}^{(1)}\right) u_{j}(t)\right) .
\end{aligned}
$$

Choose $\mu>0$ such that $\mu<\rho \delta / 2$. Since $\sum_{i=p+1}^{N} u_{i}(t) \rightarrow 0$ as $t \rightarrow \infty$, there exists $t_{3}>t_{2}$ such that

$$
\sum_{i=1}^{p} u_{i}(t)>\delta / 3 \quad \text { for } t>t_{3}
$$


Hence

$$
\sum_{j=p+1}^{N}\left(\lambda \beta_{i j}(\eta)-\kappa b_{p j}^{(1)}\right) u_{j}(t)<\mu<\rho \delta / 2 \quad \text { for } t>t_{3} .
$$

Therefore

$$
\frac{d V_{p}}{d t} \leq V_{p}\left(\kappa f_{p}(t, 0, \ldots, 0)-\lambda f_{i}(t, 0, \ldots, 0)+\sigma\right),
$$

where $\sigma=\mu-\rho \delta / 2<0$ for $t>t_{3}$. Hence

$$
V_{p}(t) \leq V_{p}\left(t_{3}\right) \exp \left\{\int_{t_{3}}^{t}\left(\kappa f_{p}(t, 0, \ldots, 0)-\lambda f_{i}(t, 0, \ldots, 0)+\sigma\right) d \tau\right\} .
$$

By the definition of $V_{p}$ we have

$$
\begin{aligned}
& u_{p}(t) \\
& \quad<\left(u_{p}^{\lambda}(t) \frac{u_{p}^{\kappa}\left(t_{3}\right)}{u_{i}^{\lambda}\left(t_{3}\right)}\right)^{1 / \kappa} \exp \left\{\frac{1}{\kappa} \int_{t_{3}}^{t}\left(\kappa f_{p}(\tau, 0, \ldots, 0)-\lambda f_{i}(\tau, 0, \ldots, 0)-\sigma\right) d \tau\right\}
\end{aligned}
$$

for $t>t_{3}$. By Lemma 4(i) there exists $R>0$ such that $u_{j}(t)<R$. Hence

$$
u_{p}(t)<D \exp \left\{\frac{1}{\kappa} \int_{t_{3}}^{t}\left(\kappa f_{p}(\tau, 0, \ldots, 0)-\lambda f_{i}(\tau, 0, \ldots, 0)-\sigma\right) d \tau\right\}
$$

for $t>t_{3}$, where

$$
D=\left(R^{\lambda} \frac{u_{p}^{\kappa}\left(t_{3}\right)}{u_{i}^{\lambda}\left(t_{3}\right)}\right)^{1 / \kappa}
$$

Similarly to $1.13-1.15$ we show that

$$
\lim _{t \rightarrow \infty} \int_{t_{3}}^{t}\left(\kappa f_{p}(\tau, 0, \ldots, 0)-\lambda f_{i}(\tau, 0, \ldots, 0)-\sigma\right) d \tau=-\infty .
$$

By (1.22) it now follows that $u_{p}(t) \rightarrow 0$ as $t \rightarrow \infty$.

Lemma 7. Any positive solution of the equation

$$
U_{1}^{\prime}(t)=U_{1}(t) f_{1}\left(t, U_{1}(t), 0, \ldots, 0\right)
$$

is defined on $[0, \infty)$, bounded above and below by positive constants, and globally attractive.

Proof. By assumption (5) we have

$$
U_{1}^{\prime}(t) \leq U_{1}(t)\left(f_{1}(t, 0, \ldots, 0)-b_{11}^{(1)} U_{1}(t)\right) .
$$

From assumptions (3) and (5) it follows that

$$
\begin{aligned}
U_{1}(t)\left(a_{1}^{(1)}-b_{11}^{(1)} U_{1}(t)\right) & \leq U_{1}(t)\left(f_{1}(t, 0, \ldots, 0)-b_{1}^{(1)} U_{1}(t)\right) \\
& \leq U_{1}(t)\left(a_{1}^{(2)}-b_{11}^{(1)} U_{1}(t)\right) .
\end{aligned}
$$


Let $c_{1}$ and $d_{1}$ be positive numbers such that $0 \leq c_{1}<a_{1}^{(1)} / b_{11}^{(1)}$ and $d_{1}>$ $a_{1}^{(2)} / b_{11}^{(1)}$. The theory of differential inequalities shows that there exists $T>0$ such that

$$
c_{1} \leq U_{1}(t) \leq d_{1} \quad \text { for } t \geq T .
$$

Now we prove attractivity of 1.23 .

Suppose that $\tilde{U}_{1}(t), \tilde{V}_{1}(t)$ are any two positive solutions of 1.23$)$. Then

$$
\begin{aligned}
& \tilde{U}_{1}^{\prime}(t)=\tilde{U}_{1}(t) f_{1}\left(t, \tilde{U}_{1}, 0, \ldots, 0\right), \\
& \tilde{V}_{1}^{\prime}(t)=\tilde{V}_{1}(t) f_{1}\left(t, \tilde{V}_{1}, 0, \ldots, 0\right) .
\end{aligned}
$$

For simplicity, assume $\tilde{U}_{1}(0)>\tilde{V}_{1}(0)$. Consequently, $\tilde{U}_{1}(t)>\tilde{V}_{1}(t)$ for all $t \geq 0$. Let

$$
\Theta(t)=\ln \frac{\tilde{U}_{1}(t)}{\tilde{V}_{1}(t)} .
$$

Then

$$
\Theta^{\prime}(t)=\frac{\tilde{U}_{1}^{\prime}(t)}{\tilde{U}_{1}(t)}-\frac{\tilde{V}_{1}^{\prime}(t)}{\tilde{V}_{1}(t)} .
$$

By 1.24 and 1.25,

$$
\Theta^{\prime}(t)=\frac{\tilde{U}_{1}^{\prime}(t)}{\tilde{U}_{1}(t)}-\frac{\tilde{V}_{1}^{\prime}(t)}{\tilde{V}_{1}(t)}=f_{1}\left(t, \tilde{U}_{1}(t), 0, \ldots, 0\right)-f_{1}\left(t, \tilde{V}_{1}(t), 0, \ldots, 0\right),
$$

and assumptions (4) and (5) yield

$$
\Theta^{\prime}(t) \leq-b_{11}^{(1)}\left(\tilde{U}_{1}(t)-\tilde{V}_{1}(t)\right)
$$

Since $\underline{\nu} \leq \tilde{U}_{1}(t) \leq \bar{\nu}$ and $\underline{\nu} \leq \tilde{V}_{1}(t) \leq \bar{\nu}$, using the mean value theorem we find that for $t>0$,

$$
\frac{1}{\bar{\nu}}\left(\tilde{U}_{1}(t)-\tilde{V}_{1}(t)\right) \leq \ln \left(\frac{\tilde{U}_{1}(t)}{\tilde{V}_{1}(t)}\right) \leq \frac{1}{\underline{\nu}}\left(\tilde{U}_{1}(t)-\tilde{V}_{1}(t)\right) .
$$

Therefore

$$
\tilde{U}_{1}(t)-\tilde{V}_{1}(t) \geq \underline{\nu} \ln \left(\frac{\tilde{U}_{1}(t)}{\tilde{V}_{1}(t)}\right)
$$

Hence

$$
\Theta^{\prime}(t) \leq-b_{11}^{(1)}(\eta) \underline{\nu} \Theta(t) \text {. }
$$

So

$$
0 \leq \Theta(t) \leq e^{-b_{11}^{(1)} \underline{\nu} t} \Theta(0) \text { for } t>0 .
$$

By 1.26), $\Theta(t) \rightarrow 0$ as $t \rightarrow \infty$. By the definition of $\Theta$ it follows that 1.23. is globally attractive.

Fix a positive solution $U_{1}^{*}(t)$ of the equation

$$
U_{1}^{\prime}(t)=U_{1}(t)\left(f_{1}\left(t, U_{1}(t), 0, \ldots, 0\right)\right) .
$$


THEOREM 2. If $u(t)=\left(u_{1}(t), \ldots, u_{N}(t)\right)$ is a positive solution of (1.1) then $u_{1}(t)-U_{1}^{*}(t) \rightarrow 0$ as $t \rightarrow \infty$.

Proof. Let $u(t)=\left(u_{1}(t), \ldots, u_{N}(t)\right)$ be a positive solution of system (1.1). Let $U_{1}(t)$ be the solution of the equation

$$
U_{1}^{\prime}(t)=U_{1}(t)\left(f_{1}\left(t, U_{1}(t), 0, \ldots, 0\right)\right)
$$

satisfying the initial condition $U_{1}(0)=u_{1}(0)$. Then by the comparison principle

$$
u_{1}(t) \leq U_{1}(t) \quad \text { for } t \geq 0, i=1, \ldots, N .
$$

By assumption (5) and by the mean value theorem,

$$
u_{1}^{\prime}(t) \leq u_{1}(t)\left(f_{1}\left(t, u_{1}(t), 0, \ldots, 0\right)-\sum_{j=2}^{N} b_{1 j}^{(1)} u_{j}(t)\right) .
$$

Let

$$
V:=\ln \left(\frac{u_{1}(t)}{U_{1}(t)}\right)
$$

Hence

$$
\frac{d}{d t}\left(\ln \frac{u_{1}(t)}{U_{1}(t)}\right)=\frac{u_{1}^{\prime}(t)}{u_{1}(t)}-\frac{U_{1}^{\prime}(t)}{U_{1}(t)}
$$

Therefore by (2.1),

$$
\frac{U_{1}^{\prime}(t)}{U_{1}(t)}=f_{1}\left(t, U_{1}(t), 0, \ldots, 0\right)
$$

By 2.2,

$$
\frac{u_{1}^{\prime}(t)}{u_{1}(t)} \leq f_{1}\left(t, u_{1}(t), 0, \ldots, 0\right)-\sum_{j=2}^{N} b_{1 j}^{(1)} u_{j}(t)
$$

Hence

$$
\frac{d V}{d t} \leq f_{1}\left(t, u_{1}(t), 0, \ldots, 0\right)-f_{1}\left(t, U_{1}(t), 0, \ldots, 0\right)-\sum_{j=2}^{N} b_{1 j}^{(1)} u_{j}(t) .
$$

By the Lagrange theorem and assumption (4),

(2.4) $f_{1}\left(t, U_{1}(t), 0, \ldots, 0\right)-f_{1}\left(t, u_{1}(t), 0, \ldots, 0\right) \geq-\beta_{11}(\eta)\left(U_{1}(t)-u_{1}(t)\right)$ for $t>t_{1}$. Therefore

$$
\frac{d V}{d t} \leq \beta_{11}(\eta)\left(U_{1}(t)-u_{1}(t)\right)-\sum_{j=2}^{N} b_{1 j}^{(1)} u_{j}(t) .
$$

Since $\sum_{i=1}^{N} u_{i}(t) \geq \delta / 2$ for $t>t_{2}$ and $\sum_{i=2}^{N} u_{i}(t) \rightarrow 0$ exponentially as $t \rightarrow \infty$ it follows that

$$
u_{1}(t)>\delta / 2 \quad \text { for } t>t_{2} .
$$


Hence and by Lemma 4(ii),

$$
\delta / 2 \leq u_{1}(t) \leq R \quad \text { for } t>t_{2} .
$$

Using the mean value theorem we see that for $t>0$,

$$
\frac{1}{R}\left(u_{1}(t)-U_{1}(t)\right) \leq \ln \left(\frac{u_{1}(t)}{U_{1}(t)}\right) \leq \frac{2}{\delta}\left(u_{1}(t)-U_{1}(t)\right) .
$$

Therefore

$$
U_{1}(t)-u_{1}(t) \leq-R \ln \left(u_{1}(t)-U_{1}(t)\right) .
$$

Hence

$$
\begin{aligned}
\frac{d V}{d t} & \leq-\beta_{11}(\eta) R \ln \left(u_{1}(t)-U_{1}(t)\right)-\sum_{j=2}^{N} b_{1 j}^{(1)} u_{j}(t) \\
& \leq-\beta_{11}(\eta) R V(t)-\sum_{j=2}^{N} b_{1 j}^{(1)} u_{j}(t) \leq-\phi V(t)+g(t)
\end{aligned}
$$

where

$$
g(t)=-\sum_{j=2}^{N} b_{1 j}^{(1)} u_{j}(t) \text { and } \phi=\beta_{11}(\eta) R
$$

Hence

$$
V(t) \leq e^{-\phi\left(t-t_{2}\right)}\left(\int_{t}^{t_{2}} g(\tau) e^{\phi\left(\tau-t_{2}\right)} d \tau+V\left(t_{2}\right)\right) .
$$

Since $g(t) \rightarrow 0$ as $t \rightarrow \infty$ it is easy to prove that $V(t) \rightarrow 0$ as $t \rightarrow \infty$. Hence and by the definition of $V$ it follows that $u_{1}(t)-U_{1}(t) \rightarrow 0$. Since $(1.23)$ is globally attractive, we conclude that $u_{1}(t)-U_{1}^{*}(t) \rightarrow 0$.

Acknowledgements. We thank the anonymous referee for his/her helpful remarks.

\section{References}

[1] S. Ahmad, On the nonautonomous Volterra-Lotka competition equations, Proc. Amer. Math. Soc. 117 (1993), 199-204.

[2] S. Ahmad and A. C. Lazer, Average conditions for global asymptotic stability in a nonautonomous Lotka-Volterra system, Nonlinear Anal. 40 (2000), 37-49.

[3] S. Ahmad and F. Montes de Oca, Extinction in nonautonomous T-periodic competitive Lotka-Volterra system, Appl. Math. Comput. 90 (1998), 155-166.

[4] B. D. Coleman, Nonautonomous logistic equations as models of the adjustment of populations to environmental change, Math. Biosci. 45 (1979), 159-173.

[5] K. Gopalsamy, Global asymptotic stability in a periodic Lotka-Volterra system, J. Austral. Math. Soc. Ser. B 27 (1986), 66-72.

[6] -, Global asymptotic stability in a periodic Lotka-Volterra system, ibid. 27 (1986), 346-360. 
[7] F. Montes de Oca and M. L. Zeeman, Extinction in nonautonomous competitive Lotka-Volterra systems, Proc. Amer. Math. Soc. 124 (1996), 3677-3687.

[8] J. Pętela, Average conditions for Kolmogorov systems, Appl. Math. Comput. 215 (2009), 481-494.

[9] A. Tineo and C. Alvarez, A different consideration about the globally asymptotically stable solution of the periodic n-competing species problem, J. Math. Anal. Appl. 159 (1991), 44-50.

Joanna Pętela

Institute of Mathematics and Computer Science

Wrocław University of Technology

Wybrzeże Wyspiańskiego 27

50-370 Wrocław, Poland

E-mail: joanna.petela@pwr.wroc.pl

Received on 5.5.2009;

revised version on 22.3.2010 
